\section{CONGENITAL OCCLUSION OF THE DUODENUM}

BY

S. GLASER, F.R.C.S.

Honorary Assistant Surgeon, Royal United Hospital, Bath

Duodenal obstruction in the newborn is a relatively rare lesion, though numbers of cases have been reported in the literature. The following two cases are considered of sufficient interest to merit recording.

\section{Case 1}

A female child, aged 10 months, was admitted to hospital because of persistent vomiting. The mother stated that the patient had suffered from projectile vomiting shortly after birth until she was three months old. (The history obtained from the mother about this phase was difficult to interpret. Later inquiries from the family doctor revealed that he had treated the child for pylorospasm, as there was projectile vomiting but no tumour, and that she had made slow but definite progress with medical treatment.) After the age of 3 months the vomiting had stopped and she had progressed satisfactorily. While away on holiday the child had started vomiting again, and for the last three days all feeds had been brought up. The vomiting was projectile, effortless, and very forceful. The child had become very ill and the parents had terminated their holiday and rushed her home. The mother told the ward sister that the vomit had at first contained some wool which she thought might have been plucked from a shawl.

Examination revealed a desperately ill, apathetic, dehydrated child with lax skin and sunken eyes. The abdomen was fallen in and scaphoid. Peristalsis was not seen, but a typical projectile vomit was observed. A tumour 1 in. $(2.5 \mathrm{~cm}$.) long was felt in the region of the pylorus. This disappeared under the fingers but was felt once more. A provisional diagnosis of pyloric stenosis was made and saline was given rectally. Next morning the general condition was slightly improved, but she was still vomiting anything given by mouth and was extremely ill. She was seen in consultation with a senior colleague. Peristalsis could not be seen and no lump could be felt, and it was decided to wait. A few hours later the child died.

Necropsy revealed a large empty stomach. The proximal half of the duodenum was very distended, with thickened and hypertrophied musculature. There was an almost complete septum across the lumen of the bowel, causing the obstruction. The septum was just proximal to the opening of the bile duct. which was running into the septum. The small aperture was plugged by a tangle of threads.

A sister born later has been seen since with a similar history of vomiting soon after birth, but a barium meal revealed no obstructive lesion.

\section{Case 2}

A female child, aged 9 days, was sent in with a diagnosis of pyloric stenosis. The history obtained from the father was that he thought the child had been all right on the first two days. but he was uncertain. He was sure, however, that from the third day she had vomited every feed, even when changed to glucosewater. The vomited material was and had been bile-stained. During the last three days vomits had become projectile in character. The bowels had not been open for six days, but before that meconium had been passed normally. The child was healthy, very hungry, and fed well, but she was losing weight. Of two older sisters, one had vomited for a few days after birth.

On examination the child was found to be in surprisingly good condition, slightly but not severely dehydrated. No lump could be felt in the abdomen. After a test-feed a wave of gastric peristalsis was observed and a bile-stained projectile vomit occurred. A diagnosis of congenital duodenal stenosis was made and consultation sought with a paediatrician. $\mathrm{He}$ agreed with the diagnosis and urged immediate surgery because of the good general condition.

Under open ether anaesthesia the abdomen was explored through a right rectus muscle splitting incision. The duodenum was found to be obstructed across its secord part by a large band. The caecum was in the region of the spleen and the small gut occupied the right part of the abdomen, the ileum entering the caecum from the right. There was no duodenojejunal flexure, the duodenum con inuing on direct into the jejunum without any bend. The constricting band appeared to contain an artery, and it was thought wiser to do a shortcircuit than to divide the band. An anterior gastro-jejunostomy was performed, making a stoma abo dt 1 in. $(2.5 \mathrm{~cm}$.) long. The abdomen was closed without drainage and without making further anatomical investigations.

Post-operatively the patient's feeding was supervised by the paediatrician; it followed roughly the same routine as in the case of a congenital hypertrophic pyloric stenosis. Occasional non-projectile vomiting occurred during the first four days, but the child fed well and her bowels opened. After the fourth day she ceased vomiting and began to gain weight. A week later she was feeding well without vomiting, the bowels were open normally, and she was gaining weight. The wound was well healed and the sutures were removed. When seen again two months later she looked puny and had not been putting on weight well. There had been some difficulty with feeding, but that was improving. Her doctor wrote eight months later that the child, then 10 months old, was eating well and putting on weight. She then weighed $16 \mathrm{lb}$. $(7.26 \mathrm{~kg}$.).

\section{Discussion}

An excellent review of the whole subject, with four case histories, has been published by Forshall (1947), and the problem is discussed in detail with a review of most of the literature. Following Ladd, cases are classified in two groups, depending on whether the obstruction is extrinsic or intrinsic. In the first group the obstruction is usually due to some abnormality of intestinal rotation, the distal duodenum being compressed by abnormal peritoneal bands or there being a volvulus of the midgut loop. The anatomical abnormality commonly found in these cases is the colon in the left abdomen, with the ileum entering the caecum from the right. In the second type of obstruction the cause may be a complete stenosis of the duodenum, a narrowing of its lumen, or a septum partially or completely occluding it. This usually occurs in the region of the ampulla of Vater. Keith (1933) points out that the lumen in this region of the duodenum is normally occluded by proliferating epithelium during the fifth and sixth weeks of embryonic life, when the liver and pancreatic buds are growing out, and that in cases of congenital occlusion of the duodenum the epithelium becomes organized instead of being absorbed.

In both groups the clinical picture is similar and characteristic. Vomiting is the main symptom and starts within a day or two of birth. It is usually bile-stained, as in most cases the obstruction is below the main bile duct. The child becomes dehydrated rapidly and loses weight, and if untreated dies of inanition. Each of the above cases illustrates one of these varieties.

In Case 1 the main interest lies in the length of survival of the child. The aperture in the septum across the duodenum was large enough to take a fluid diet and the early stages of a mixed diet. The obstruction was caused finally by the blocking of the small hole by some cotton or wool. The possibility arises that many cases are undiagnosed, and it is striking that great numbers are reported from Boston (Mass.), where there is a very keen interest in congenital lesions. Many may die unrecognized, and without postmortem examination may be wrongly certified. Furthermore, this case of incomplete septum shows how careful medical treatment carried the child over the initial obstructive stage. Some cases, like this one, survive the early stages and become obstructed later. Forshall quotes Seidlin's case of a child who died of obstruction at the age of $2 \frac{1}{2}$ years, after eating canned corn. Balfour (1932) records the case of 
a patient aged 12 on whom he operated for increasing obstruction with vomiting, and in whom the duodenum was two-thirds closed.

In Case 2 the obstruction was of the extrinsic variety and showed the main characteristic features-i.e., bile-stained vomiting coming on soon after birth, loss of weight, and dehydration. At operation the colon was on the left side of the abdomen with the caecum in the region of the spleen, while the small gut was on the right side. The main interest, however, lies in the fact that the child survived the operation of gastro-jejunostomy. Forshall tabulates in her paper the previously recorded survivals after an anastomosis and reports two further cases, making in all a total of 32. Case 2 adds another to the small list.

\section{Summary}

Two cases of duodenal obstruction in the newborn are described, one of which survived gastro-jejunostomy.

The syndrome of congenital occlusion of the duodenum is clear-cut, and though rare it should be recognized when it occurs and should be diagnosed early. This is important, as the condition is amenable to surgical intervention, and, contrary to general belief, the very young infant stands up extremely well to the required major surgery.

REFERENCES

Balfour, D. C. (1932). Collected Papers of the Mayo Clinic, 24,

Forshail, I. (1947). Brit. J. Surg., 35, 58.

Keith, A. (1933). Human Embryology and Morphology. London.

\section{URETERIC CALCULUS CAUSING ANURIA} BY

NESTOR J. S. NATHAN, M.R.C.S., D.P.H., D.R.C.O.G. AND

CYRIL JOSEPHS, M.B., B.S., D.C.H.

(From the Staincliffe General Hospital, Dewsbury)

Anuria due to mechanical obstruction is an uncommon phenomenon. In the following case the patient had absolute anuria, which was found to be due to a calculus blocking the ureter just below the pelvis of a solitary kidney. He made a complete recovery after removal of the calculus.

\section{Case Report}

The patient, a man aged 63 , had been gassed during the 1914-18 war and subsequently had had bronchitis. In 1936 a perforated duodenal ulcer had been oversewn. He was fit until 1946, when frequency and difficulty of micturition began. In June, 1946, a transurethral prostatectomy was performed. $\mathrm{He}$ remained unwell and lost a considerable amount of weight. He was admitted to hospital on Dec. 9, 1946, his general condition being very poor. The urine contained pus and grew a culture of $B$. coli. An intravenous pyelography showed a nonfunctioning right kidney. A course of su'phathiazole combined with penicillin had no effect.

On Dec. 20 the right kidney was explored by Mr. E. R. Flint. There was a large pyelonephrosis, but no obvious cause could be found. The right kidney was removed. After operation the patient made an excellent recovery, the urine cleared up, and he gained weight rapidly.

On Aug. 19, 1947, he was readmitted complaining of pain in his left loin and anuria. On catheterization only $1 \mathrm{oz}$. $(28 \mathrm{ml}$.) of urine was obtained. The urine was normal. The blood urea was $279 \mathrm{mg}$. per $100 \mathrm{ml}$. of blood. Anuria persisted for seven days in spite of intravenous $4.285 \%$ sodium sulphate solution, fluids, hot packs to the loin, light baths, pilocarpine, and cupping. Cystoscopy was carried out and intravenous indigo carmine given. There was no secretion from the left kidney.

A second operation was performed by Mr. E. R. Flint on Aug. 26. The left kidney was explored and freed from its bed. A stone was palpable in the ureter 3 in. $(7.5 \mathrm{~cm}$.) below the renal pelvis. An incision was made in the ureter. There was a profuse discharge of pus, and a stone the size of a plum-stone impacted in the left ureter was removed. The ureter was drained and a superficial drain was also inserted into the wound. Eighteen hours after his return from the theatre $6 \mathrm{oz} .(170 \mathrm{ml}$.) of urine was passed normally. Urine drained freely from the ureteric drain, but each day the amount of urine passed normally increased. The ureteric drain was removed on the fifth day; the wound healed, and no residual sinus occurred. The patient was discharged on Sept. 29, 1947, perfectly fit and passing urine normally.

We are indebted to Mr. E. R. Flint for permission to publish this report.

\section{Medical Memoranda}

\section{Unusual Case of Twins}

The following case is unusual enough to merit publication.

\section{CASE History}

A married woman aged 23 was referred to us on May 22, 1947, as a possible case of twins. She had had a normal delivery of a living healthy child on April 7, 1946. Her previous medical history revealed nothing of note. On examination a severe degree of anaemia was present. The abdomen measured 38 in. $(96 \mathrm{~cm}$.). Palpation of the abdomen suggested the presence of twins. $X$-ray examination next day confirmed the diagnosis and showed that both foetuses were presenting by the breech.

The patient later stated that after the $x$-ray examination she went home and spent most of the afternoon scrubbing her kitchen floor. She was sick and vomited once during the night. Next day she was uncomfortable all day, complaining of a dragging pain on the right side of the abdomen. She retired to bed at $8 \mathrm{p} . \mathrm{m}$. and slept until about 5.30 a.m., when she was awakened by the onset of labour. The pains were frequent and strong. The midwife did not arrive until about 7 a.m., by which time the first child had been born (at 6.15 a.m.) by the vertex. A neighbour who was present at the time is quite certain about this. The midwife separated the infant and, because of a mass in the vagina, sent for us. By the time we arrived, about 8 a.m., a placenta had been expelled, but the cord led into the uterus. The cord of the already born infant also led into the uterus.

Vaginal examination revealed the second foetus presenting by the vertex in the L.O.P. position. Manual rotation was easily carried out, and two more pains sufficed to expel the infant-dead. The placenta of the living twin was easily expressed some 15 minutes later. At no stage during labour or after did any undue haemorrhage occur, which was fortunate considering the degree of anaemia present

Both placentae appeared healthy. The opening in the first bag of membranes delivered (that of the second child) was close to the edge: that of the second nearly central. The first child weighed $5 \frac{1}{2} \mathrm{lb} .(2.5$ $\mathrm{kg}$.), the second $6 \frac{3}{4} \mathrm{lb}$. $(3.1 \mathrm{~kg}$.). Both were males. The mother made an uninterrupted recovery.

\section{COMMENT}

The outstanding points in this case seem to be : (1) Spontaneous version so near to the onset of labour-less than 43 hours. The events of the day before delivery suggest that version occurred on that day. (2) The unusual sequence of events during labour : foetus A, placenta B, foetus B, placenta A. Such an occurrence must be very rare. We can find no similar case in modern textbooks. Meigs (1856) mentions a case in these words: "I saw a twin labour in which the first child pushed the placenta of its brother before it into the world." $\mathrm{He}$ gives no further details and we are left in doubt whether this case was exactly similar to ours. Smellie (Case 411) describes a case in which the membranes of the second child came down in advance of the head and membranes of the first child (McClintock, 1876). Commenting on this, McClintock states that he never met with such an anomaly, and adds: "Were it not recorded by such a careful and accurate observer as William Smellie, I would feel inclined to doubt its actual occurrence."

$$
\begin{aligned}
& \text { J. H. Young, M.B., Ch.B., D.R.C.O.G. } \\
& \text { F. E. Cull, M.B., Ch.B. } \\
& \text { References }
\end{aligned}
$$

McClintock, A. H., Editor (1876). Smellie's Treatise on the Theory and Practice of Midwiferv, vol. 3, p. 202. London. Meigs, C. D. (1856). Science and Art of Obstetrics. Philadelphia. 